Supporting Information for

\title{
Mechanical Properties of Solution-Processed Small-Molecule Semiconductor Films
}

Daniel Rodriquez, ${ }^{1}$ Suchol Savagatrup, ${ }^{1}$ Eduardo Valle, ${ }^{1}$ Christopher M. Proctor, ${ }^{2}$ Caitlin McDowell, ${ }^{2}$ Guillermo C. Bazan, ${ }^{2}$ Thuc-Quyen Nguyen, ${ }^{2}$ and Darren J. Lipomi ${ }^{1 *}$

${ }^{1}$ Department of NanoEngineering, University of California, San Diego

9500 Gilman Drive, Mail Code 0448, La Jolla, CA 92093-0448

${ }^{2}$ Center for Polymers and Organic Solids, Department of Chemistry and Biochemistry, University of California Santa Barbara, CA 93106-9510

*Author to whom correspondence should be addressed: dlipomi@ucsd.edu 
Figure S1 shows the performance of small-molecule BHJs with HPI-BT as the acceptor. We did not investigate the photovoltaic performance of these devices under strain because of their low PCE values. The most efficient solar cells, those containing $\mathrm{PC}_{71} \mathrm{BM}$ as the acceptor, were not measured under strain since they cracked at very low strains. Cracking leads to failure by disconnecting the active regions from the electrodes, shorting of the electrodes through the film, or both.
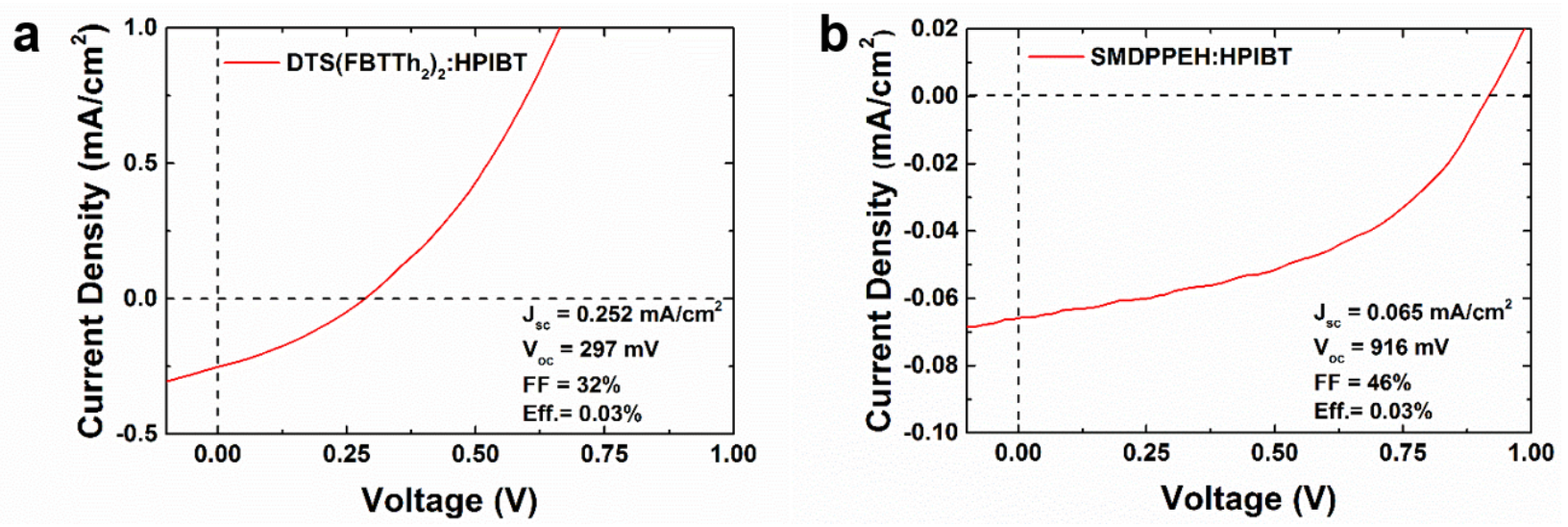

Figure S1. Photovoltaic plots of (a) DTS(FBTTh $)_{2}$ :HPI-BT and (b) SMDPPEH:HPIBT bulk heterojunction solar cells. The curves represent average values of $N=3$ devices for both (a) and (b), the inset shows the values from the best performing solar cells. 


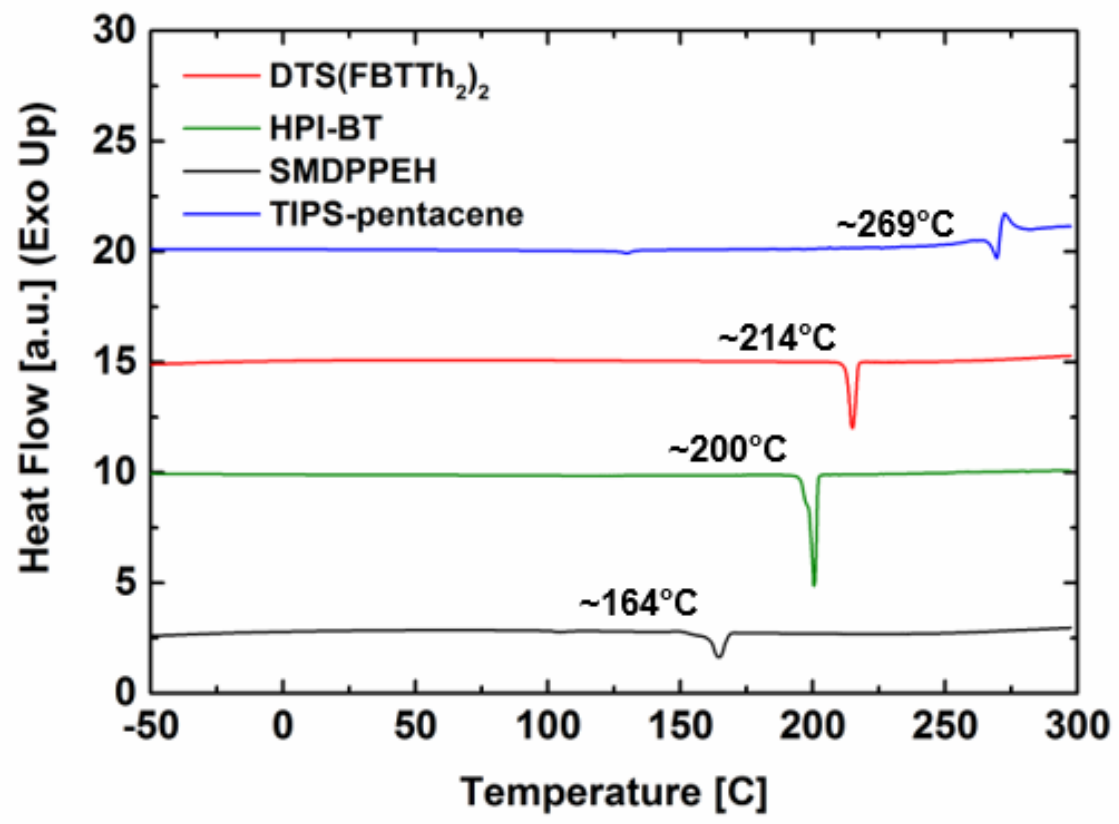

Figure S2. DSC thermograms showing the melting temperature of DTS(FBTTh $)_{2}$, SMDPPEH, TIPS-pentacene, and HPI-BT. 


\section{DTS $\left(\mathrm{FBTTh}_{2}\right)_{2}$}

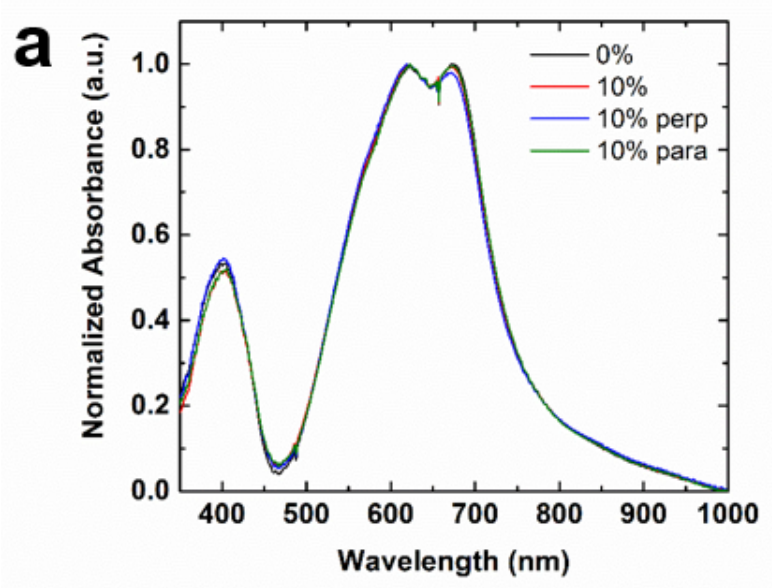

HPI-BT

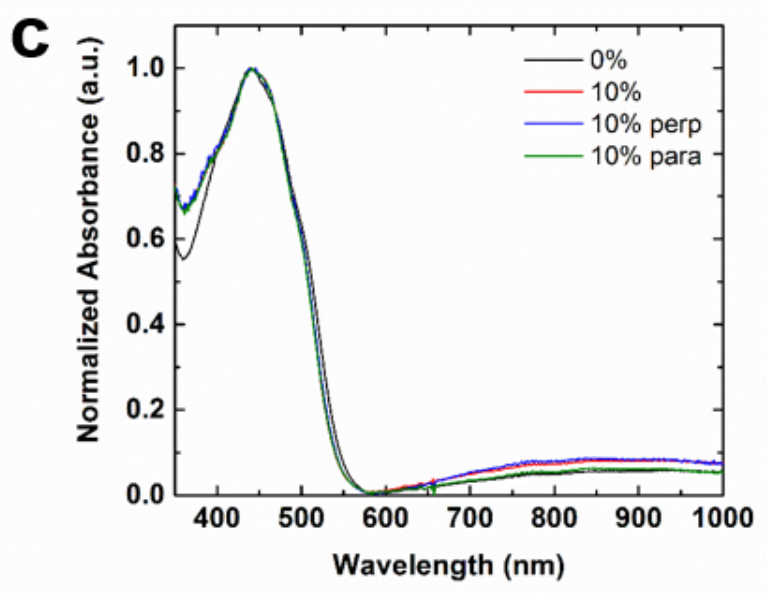

SMDPPEH

b

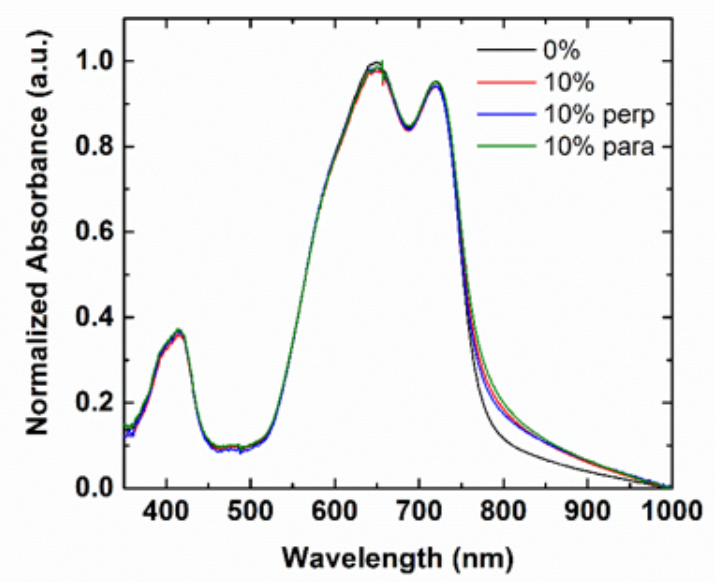

$\mathrm{PC}_{71} \mathrm{BM}$

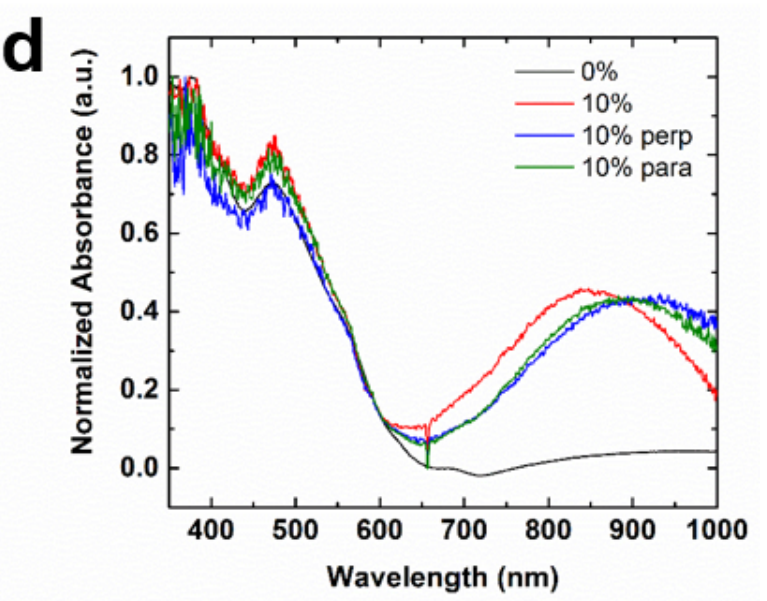

Figure S3. Absorption spectra of as-cast pure (a) DTS(FBTTh $)_{2}$, (b) SMDPPEH, (c) HPI-BT, and (d) $\mathrm{PC}_{71} \mathrm{BM}$ thin films. All films were analyzed on PDMS with a device architecture of PDMS/PEDOT:PSS/Film. Spectra was first collected from unstrained films and sequentially after being strained to $10 \%$ with no polarization and with polarization both perpendicular (perp) and parallel (para) to the axis of strain. 